\title{
La osteonecrosis asociada al tratamiento con bisfosfonatos en osteoporosis primaria. Revisión de la literatura
}

\author{
Osteonecrosis of the jaw associated with bisphosphonate \\ therapy in primary osteoporosis. Review of the literature
}

\section{A. Outeiriño-Fernández}

\section{RESUMEN}

Fundamento. El propósito de esta revisión es identificar los factores de riesgo que pueden intervenir en el desarrollo de la osteonecrosis maxilar asociada a bisfosfonatos (OMB) tras cirugía oral, en pacientes en tratamiento con bisfosfonatos (BP) por osteoporosis primaria.

Material y métodos. Con el fin de localizar las publicaciones de relevancia se llevó a cabo una búsqueda en bases de datos tanto de ámbito especializado, como general. La estrategia de búsqueda empleada fue osteonecrosis, bisfosfonatos y cirugía oral. Ésta fue limitada a estudios publicados desde el año 2006 a enero de 2012, en inglés y español realizados en humanos, adultos, tratados con bisfosfonatos por osteoporosis primaria y sometidos a cirugía dentoalveolar. Fueron incluidas revisiones sistemáticas, meta-análisis, guías de práctica clínica, ensayos clínicos aleatorizados y series de casos.

Resultados. Se seleccionaron un total de 14 artículos (series de casos), que recogían un total de 43 casos de OMB. La mandíbula y la exodoncia fueron la localización y procedimiento odontológico más frecuente en relación con las lesiones descritas. La media de edad de los pacientes fue de 71,94 años, con predilección por el sexo femenino. El alendronato fue el fármaco empleado en la mayoría de los casos y la duración media de tratamiento previa a la aparición de OMB fue de 48,25 meses.

Conclusiones. Son necesarios más estudios prospectivos y retrospectivos con un diseño adecuado para evaluar el peso real de cada uno de los factores considerados de riesgo para el desarrollo de la OMB.

Palabras clave. Osteonecrosis. Osteoporosis. Bisfosfonato. Cirugía oral.

\begin{abstract}
Background. The aim of this review was to study risk factors involved in osteonecrosis of the jaw after oral surgery in patients treated with bisphosphonates due to primary osteoporosis.

Methods. In order to find relevant related publications, we conducted a systematic search in specialized (Cochrane Database of Systematic Reviews, Trip Database) and general (Medline, Embase, Isi Web of Knowledge) bibliographical databases. The search strategy included the terms osteonecrosis, adults and oral surgery. It was limited to papers focused on human adults undergoing dentoalveolar surgery while being treated with bisphosphonates due to primary osteoporosis, which had been published in the English or Spanish languages between January 2006 and January 2012. Systematic reviews, meta-analyses, practice guidelines, randomized clinical trials and case report series were included.
\end{abstract}

Results. Fourteen papers (case report series) including 43 cases of osteonecrosis of the jaw were selected. Jaw and extraction were the most frequent localization and dental procedure related to the lesions. Mean age of patients was 71.94 years, with lesions being more frequent among women. Alendronate was the bisphosphonate used in the majority of cases, with a mean duration of treatment before osteonecrosis of the jaw appeared of 48.25 months.

Conclusions. Well-designed, prospective and retrospective studies are needed to evaluate the real weight of each of those factors considered to entail a potential risk of osteonecrosis of the jaw.

Key words. Osteonecrosis. Osteoporosis. Bisphosphonate. Oral surgery.
Odontología. Área Primaria. Xerencia de Xestión Integrada de Ferrol. SERGAS.

Recepción: 26 de septiembre de 2012

Aceptación provisional: 5 de octubre de 2012

Aceptación definitiva: 6 de noviembre de 2012

\section{Correspondencia:}

Alejandra Outeiriño Fernández

Centro de Saúde As Pontes de García Rodríguez Saude, $\mathbf{s} / \mathrm{n}$

15320 As Pontes de García Rodríguez (A Coruña)

E-mail: Alejandra.Outerino.Fernandez@sergas.es 


\section{INTRODUCCIÓN}

La osteoporosis se define como una enfermedad esquelética sistémica, caracterizada por una disminución de la masa ósea y deterioro de la microarquitectura del tejido óseo. El consiguiente aumento de su fragilidad predispone a la fractura ósea ${ }^{1}$ Establecer su verdadera prevalencia es complejo. En el caso de España, hay estudios que indican que se encuentra en torno al 35\% en mujeres mayores de 50 años, aumentando hasta un $52 \%$ en mayores de $70^{2}$

En la actualidad, aunque existen diversas alternativas para su tratamiento, los bisfosfonatos (BP) constituyen el grupo farmacológico más ampliamente utilizado ${ }^{3}$. Sin embargo, es importante resaltar que su uso terapéutico conlleva una serie de efectos adversos. A nivel óseo, cabe destacar su relación con el incremento de fracturas atípicas (subtrocánter de la diáfisis femoral), dolor músculo-esquelético o la osteonecrosis maxilar asociada a bisfosfonatos $(\mathrm{OMB})^{4}$, de localización prácticamente exclusiva a nivel de ambos maxilares.

La Asociación Americana de Cirujanos Orales y Maxilofaciales (AAOMS) (http:// www.aaoms.org), define la OMB como hueso expuesto en la región maxilofacial que persiste más de 8 semanas en pacientes en tratamiento actual o previo con BP y sin antecedentes de radioterapia a nivel de maxilares ${ }^{5}$

Los primeros casos descritos datan de $2003^{6}$ y $2004^{7}$, fecha en la que los BP ya estaban comercializados y eran utilizados en la práctica clínica diaria.

Por otro lado, los dos factores de riesgo más importantes para su desarrollo son la exposición a BP por vía intravenosa, y los procedimientos a nivel dentoalveolar (hasta en un $70 \%$ de los casos) $)^{5,8}$

Hasta la fecha, el riesgo de OMB para pacientes en tratamiento con BP intravenosos, asociados a enfermedades neoplásicas, es significativamente mayor que en pacientes en tratamiento por vía oral ${ }^{3,5,9}$. Generalmente, la administración oral es la empleada de modo habitual para el tratamiento de la osteoporosis, reservándose la vía intravenosa para casos severos, pacientes con un mal cumplimiento de la pauta por vía oral o polimedicados.
Se estima que el riesgo de desarrollar OMB en pacientes en tratamiento por osteoporosis es de 1/10.000 y 1/100.000 pacientes/tratamiento y año ${ }^{10}$, aunque en un estudio realizado ${ }^{11}$, esta frecuencia aumentaba hasta entre un $0,1-0,4 \%$ y a un 0,09 $0,34 \%$ cuando al paciente se le había realizado una exodoncia.

En la actualidad su patogénesis y el motivo por el cual únicamente afecta a ambos maxilares se desconoce y las recomendaciones para el manejo de estos pacientes se basan en opiniones de expertos ${ }^{5,8,12-16}$ No existen, por tanto, técnicas eficaces de tratamiento basadas en la evidencia, por lo que la prevención es de suma importancia.

El objetivo del estudio es revisar la literatura para identificar los factores de riesgo que pueden intervenir en el desarrollo de la OMB tras cirugía oral, en pacientes en tratamiento con BP por osteoporosis primaria.

\section{MATERIAL Y MÉTODOS}

Se llevó a cabo una revisión sistemática (RS) de la literatura.

La búsqueda de información se llevó a cabo en dos tipos de bases de datos: especializadas en RS (HTA y Cochrane) y otros documentos de ámbito especializado (NGC), y bases de datos de ámbito general (Medline, Embase, e ISI WOK). Las estrategias de búsqueda empleadas están recogidas en la tabla 1. La búsqueda se limitó a trabajos escritos en español e inglés, publicados desde el año 2006 hasta enero de 2012. Con respecto a los tipos de estudios, tan sólo se incluyeron revisiones sistemáticas, meta-análisis, guías de práctica clínica (GPC), ensayos clínicos aleatorizados (ECA) y series de casos.

Para la selección de casos, se establece como definición de $\mathrm{OMB}$, la propuesta por la AAOMS en su documento de posición más actualizado $(2009)^{5}$ Fueron incluidos casos de pacientes en tratamiento con BP por osteoporosis primaria, que hubieran sufrido osteonecrosis tras cirugía dentoalveolar, incluyéndose entre los procedimientos exodoncias, cirugía periodontal, periapical, implantológica, de regularización ósea, etc. 
Tabla 1. Bases de datos y estrategias de búsqueda

\author{
BIBLIOTECA COCHRANE PLUS (Osteonecrosis* Mandibula* Bifosfonato*):TA \\ Osteonecros* Diphosponate*:TA
}

\begin{tabular}{ll}
\hline TRIP DATABASE & (osteonecrosis AND bisphosphonates AND oral) \\
\hline MEDLINE / PUBMED & ("Osteonecrosis/chemically induced" [Mesh] OR "Osteonecrosis/ \\
prevention and control”[Mesh]) AND ("Diphosphonates/adverse \\
effects"[Mesh] OR "Diphosphonates/therapeutic use"[Mesh]) \\
AND "Oral Surgical Procedures"[Mesh]
\end{tabular}

ISI WEB OF KNOWLEDGE

Topic $=\left(\right.$ osteonecro $\left.^{*}\right)$ AND ((bisphosphonat* (surg OR EXTRACT)

Refined by: Languages $=($ ENGLISH $)$ AND Document Type $=($ ARTICLE

OR REVIEW). Timespan=2006-2012. Databases=SCI-EXPANDED.

EMBASE

bisphosphonic acid derivative/ae, dt [Adverse Drug Reaction, Drug Therapy] AND bone necrosis/et, pc [Etiology, Prevention] AND oral surgery/ AND and 2006-2012.(sa_year).Languages: english, spanish

Se excluyeron casos de pacientes en tratamiento por neoplasias con elevado riesgo de hipercalcemia y/o metástasis, pacientes inmunodeprimidos, en tratamiento con corticoesteroides o con diabetes mellitus, factores considerados de riesgo para el desarrollo de OMB.

Los resultados de las búsquedas fueron descargados en un gestor de referencias bibliográficas (Endnoteweb), con el fin de eliminar todos los duplicados existentes. De estas referencias, se analizaron el resumen de las mismas para saber si se adaptaban a los criterios definidos. En determinados casos fue necesario acceder al texto completo.

La calidad de la evidencia científica de los estudios seleccionados fue valorada según su diseño, de acuerdo con la escala de Oxford $^{17}$

\section{RESULTADOS}

La búsqueda inicial de RS ofreció un resultado $^{3}$, que recogía los casos publicados hasta el año 2006. Dicho estudio sirvió de base para nuestro trabajo, ya que se llevó a cabo una actualización de la búsqueda bibliográfica (desde octubre de 2006 en adelante). Tras la lectura de los títulos y resúmenes de los artículos, se seleccionaron un total de 51. Finalmente 14 cumplían los criterios de inclusión descritos con anterioridad (Tabla 2 ).

La revisión de los 14 artículos seleccionados (Tabla 3) aportó información sobre la edad y el sexo de un total de 43 pacientes, de los cuales 40 eran mujeres y tres varones (proporción 13:1). La edad media de los pacientes con OMB fue de 71,94 años, oscilando en un intervalo de entre 49 y 86 años.

La exodoncia fue el acto quirúrgico que con mayor frecuencia fue referido como factor relacionado con la OMB (35 casos $)^{18-28}$, seguido de la cirugía de implantes (siete casos, uno de ellos con elevación sinusal ${ }^{29-31}$, registrándose un solo caso tras la realización de una alveoloplastia ${ }^{29}$

En cuanto al tipo de fármaco, el alendronato fue el empleado con mayor frecuencia, siendo utilizado en 33 de los pacientes de forma exclusiva ${ }^{18,19,21,24-31}$ y alternándose en dos casos con la utilización de pamidronato $^{22}$ e ibandronato ${ }^{20}$. De los individuos restantes, siete recibieron risedronato ${ }^{21,23,26,31} \mathrm{y}$ uno pamidronato ${ }^{22}$. 
Tabla 2. Número de casos, nivel de evidencia y grado de recomendación de la bibliografía seleccionada.

\begin{tabular}{|c|c|c|c|c|}
\hline Bibliografía & Tipo de artículo & № Pac & N.E & G.R \\
\hline Harper RP y col ${ }^{18}$ & Informe de un caso & 1 & IV & $\mathrm{C}$ \\
\hline Kumar SKS y col ${ }^{19}$ & Serie de casos & 2 & IV & $\mathrm{C}$ \\
\hline Fantasia JE y col ${ }^{20}$ & Serie de casos & 1 & IV & $\mathrm{C}$ \\
\hline Kunchur R y col ${ }^{21}$ & Serie de casos & 5 & IV & $\mathrm{C}$ \\
\hline Alons $\mathrm{K} \mathrm{y} \mathrm{col}^{22}$ & Serie de casos & 2 & IV & $\mathrm{C}$ \\
\hline Yamaguchi $\mathrm{K}$ y col${ }^{23}$ & Informe de dos casos & 2 & IV & $\mathrm{C}$ \\
\hline Dannemann C y col${ }^{24}$ & Serie de casos & 3 & IV & $\mathrm{C}$ \\
\hline Hutchinson $\mathrm{M} \mathrm{y} \mathrm{col}^{25}$ & Serie de casos & 5 & IV & $\mathrm{C}$ \\
\hline Kwon YD y col ${ }^{26}$ & Serie de casos & 13 & IV & $\mathrm{C}$ \\
\hline Subramanian G y col ${ }^{27}$ & Revisión de series de casos & 1 & IV & $\mathrm{C}$ \\
\hline Treister NS y col${ }^{28}$ & Serie de casos & 1 & IV & $\mathrm{C}$ \\
\hline Narongroeknawin $\mathrm{P}_{\text {y col}}{ }^{29}$ & Informe de un caso. & 1 & IV & $\mathrm{C}$ \\
\hline Bedogni A y col ${ }^{30}$ & Informe de un caso & 1 & IV & $\mathrm{C}$ \\
\hline Goss A y col ${ }^{31}$ & Serie de casos & 5 & IV & $\mathrm{C}$ \\
\hline
\end{tabular}

№ Pac: número de pacientes. N.E: nivel de evidencia. G.R: grado de recomendación.

En relación con la dosificación, se recogieron datos sobre las cantidades de fármaco empleadas en un total de 20 individuos $^{18,19,23,26,27,30}$, no siendo evaluable en los casos restantes. De los pacientes a tratamiento con alendronato, 16 tenían pautado el fármaco a dosis de $70 \mathrm{mg}$ con una frecuencia semanal y un paciente recibía esta misma dosis diaria. La dosis de risedronato fue recogida en tres pacientes, dos de ellos con una posología de $2,5 \mathrm{mg}$ al día y el tercero con $35 \mathrm{mg}$ semanales. Se desconoce la dosis empleada en el tratamiento con pamidronato, así como de aquellos que recibieron respectivamente pamidronato/alendronato e ibandronato/ alendronato.

La duración media del tratamiento con BP fue de 48,25 meses, siendo en el caso del alendronato de 48,27 meses y 47,66 meses para el risedronato. El pamidronato fue utilizado por un único paciente que lo recibió durante 25,2 meses $^{22}$, mientras que para los pacientes en tratamiento con pamidronato/alendronato ${ }^{22}$ e ibandronato/ alendronato ${ }^{20}$ la duración fue de 72 meses y más de 168 meses respectivamente.
La localización predominante de las lesiones fue a nivel mandibular con un total de 25 casos de osteonecrosis ${ }^{18-22,23,25-27,30,31}$ En maxilar superior se localizaron ocho ca$\operatorname{sos}^{23,25,26,29,31}$, uno de ellos con presentación bilateral ${ }^{29}$ y tres pacientes presentaron lesiones ubicadas en ambos maxilares ${ }^{26,28}$. En los siete casos restantes, no se encontró información sobre la localización de la $\mathrm{OMB}^{21}$

En los artículos revisados, los datos clínicos recogidos no permitieron establecer el estadio de los casos de OMB, según los criterios de la AAOMS, en 16 de las lesiones evaluadas. En los casos restantes, la distribución fue la siguiente: ocho casos presentaron estadio $0^{20,23,25}$, ocho estadio $\mathrm{I}^{26,29}$, nueve estadio II $^{24,26-28}$ y dos casos estadio III ${ }^{26,31}$. En los pacientes que presentaron localizaciones mixtas las lesiones en uno de los casos se encontraban en idéntico estadio (II) ${ }^{28}$, y en los otros dos casos, la evolución de las lesiones fue diferente: en el paciente con dos localizaciones una en cada maxilar, el estadio a nivel mandibular fue III, y I a nivel de maxilar superior ${ }^{26}$. El paciente restante presentaba dos lesiones mandibulares, con estadios I y II y la lesión maxilar con estadio $\mathrm{I}^{26}$ 
Tabla 3. Características principales de los estudios: datos correspondientes al número de casos registrados por cada autor y en cuántos de estos casos se registró la categoría seleccionada

\begin{tabular}{|c|c|c|c|c|c|c|c|c|c|c|c|}
\hline \multirow{2}{*}{ Autores } & \multirow{2}{*}{ Casos } & \multicolumn{10}{|c|}{ Número de casos que registran información sobre la variable } \\
\hline & & Edad & Sexo & Bp & Dosis & Duración & Admon & Clínica & Localización & $\mathrm{H}^{\mathrm{a}}$ Médica & Evolución \\
\hline Harper y col ${ }^{18}$ & 1 & 1 & 1 & 1 & 1 & $\mathrm{X}$ & 1 & 1 & 1 & 1 & 1 \\
\hline Kumar y col ${ }^{19}$ & 2 & 2 & 2 & 2 & 2 & 2 & 2 & $\mathrm{X}$ & 2 & 2 & $\mathrm{X}$ \\
\hline Fantasía $^{20}$ & 1 & 1 & 1 & 1 & $\mathrm{X}$ & 1 & 1 & 1 & 1 & $\mathrm{X}$ & $\mathrm{X}$ \\
\hline Kunchur y $\operatorname{col}^{21}$ & 5 & 5 & 5 & 5 & $\mathrm{X}$ & 5 & 4 & 5 & 1 & $\mathrm{X}$ & 1 \\
\hline Alons y col$^{22}$ & 2 & 2 & 2 & 2 & $\mathrm{X}$ & 2 & 2 & 2 & 2 & $\mathrm{X}$ & 2 \\
\hline Yamaguchi y col$^{23}$ & 2 & 2 & 2 & 2 & 2 & 2 & $\mathrm{X}$ & 2 & 2 & 2 & 2 \\
\hline Dannemann y col ${ }^{24}$ & 3 & 3 & 3 & 3 & $\mathrm{X}$ & 3 & $\mathrm{X}$ & 3 & $\mathrm{X}$ & $\mathrm{X}$ & 3 \\
\hline Hutchinson y col' ${ }^{25}$ & 5 & 5 & 5 & 5 & $\mathrm{X}$ & 5 & 5 & 5 & 5 & 5 & $\mathrm{X}$ \\
\hline Known y col' ${ }^{26}$ & 13 & 13 & 13 & 13 & 13 & 13 & $\mathrm{X}$ & 13 & 13 & 13 & $\mathrm{X}$ \\
\hline Subramanian y $\operatorname{col}^{27}$ & 1 & 1 & 1 & 1 & $\mathrm{X}$ & $\mathrm{X}$ & $\mathrm{X}$ & 1 & 1 & 1 & 1 \\
\hline Treister y col $^{28}$ & 1 & 1 & 1 & 1 & $\mathrm{X}$ & $\mathrm{X}$ & $\mathrm{X}$ & 1 & 1 & $\mathrm{X}$ & $\mathrm{X}$ \\
\hline Narongroeknawin y col' ${ }^{29}$ & 1 & 1 & 1 & 1 & $\mathrm{X}$ & 1 & 1 & 1 & 1 & 1 & 1 \\
\hline Bedogni y col $^{30}$ & 1 & 1 & 1 & 1 & $\mathrm{X}$ & 1 & 1 & 1 & 1 & $\mathrm{X}$ & 1 \\
\hline Goss y $\operatorname{col}^{31}$ & 5 & 5 & 5 & 5 & $\mathrm{X}$ & 5 & $\mathrm{X}$ & $\mathrm{X}$ & 5 & 5 & 5 \\
\hline TOTAL & 43 & 43 & 43 & 43 & 10 & 40 & 17 & 36 & 36 & 30 & 17 \\
\hline
\end{tabular}

BP: Tipo de bisfosfonato. Admon: incluye administración vía oral o intravenosa. Clínica: incluye descripción de signos y síntomas de las lesiones y estadios clínicos. $\mathrm{H}^{\mathrm{a}}$ médica: historia médica: incluye comorbilidades, tratamientos concomitantes. X: no evaluable

La evolución de las lesiones fue registrada en 17 pacientes s,21-24,27,29-31 $^{18}$ de tal modo que se produjo la resolución completa en 16 casos y en uno de ellos se produjo alivio de la sintomatología ${ }^{30}$

Los resultados en relación a las pruebas complementarias utilizadas como apoyo al diagnóstico clínico de la OMB fueron los siguientes:

- Estudios radiográficos (ortopantomografía y periapicales): dada la afectación ósea que produce la OMB, suelen ser la primera opción de apoyo al diagnóstico, aunque no se especifica su realización en el trabajo de Goss y col $^{31}$ y en uno de los casos presentados por Yamaguchi y $\mathrm{col}^{23}$. En los casos restantes, estas pruebas de imagen fueron empleadas en todos los pacientes, siendo los hallazgos más frecuentes la osteoesclerosis, así como lesiones líticas, secuestros óseos, persistencia de alveolos post-exodoncia, lámina dura engrosada y defectos a nivel cortical.

- Tomografía computerizada (TC y TC de haz cónico): fue empleada en 28 de los casos analizados. En las restantes publicaciones no se especifica su uso o en qué casos fue utilizada ${ }^{19}$. En la mayoría de los casos se confirmaron los hallazgos radiográficos y en una publicación condujo al diagnóstico de una comunicación sinusal asintomática y un secuestro ${ }^{28}$

- Resonancia magnética: consta su utilización en únicamente seis de los 43 casos recogidos ${ }^{18,22,24}$

- Gammagrafía ósea: realizada en seis casos, aumentando la captación en el área de hueso lesionada ${ }^{18,22,23,30}$

- Estudios microbiológicos: realizados en seis casos, en los cuales el componente infeccioso estaba presen- 
te $\mathrm{e}^{18,22,24}$, encontrándose presente el Actinomyces en todos ellos excepto en un caso ${ }^{18}$

- Biopsia: fue realizada en siete de los casos en los que los hallazgos confirman la existencia de proceso inflamatorio y hueso necrótico ${ }^{18,22,29,30}$

- Telopéptido C-terminal del colágeno tipo I en suero (CTXs): se analizó en 18 de los $43 \operatorname{casos}^{21,26}$, de los cuales cinco presentaban valores inferiores a $100 \mathrm{pg} / \mathrm{ml}$, siete casos con valores de entre 100 y $150 \mathrm{pg} / \mathrm{ml}$ y los seis restantes con cifras superiores a 150 $\mathrm{mg} / \mathrm{ml}$ (tres de ellos por encima de $200 \mathrm{pg} / \mathrm{ml})$.

Tabla 4. Principales resultados obtenidos en relación con los 43 casos registrados

\begin{tabular}{|ll|}
\hline Edad (años) & 71,94 (49-86 años) \\
\hline Sexo & Hombres: 3 \\
& Mujeres: 40 \\
Fármaco empleado & Alendronato: 33 \\
& $\begin{array}{l}\text { Risedronato: } 7 \\
\text { Pamidronato: } 1 \\
\text { Terapia combinada: } 2\end{array}$ \\
\hline $\begin{array}{l}\text { Duración media del } \\
\text { tratamiento (meses) }\end{array}$ & 48.25 \\
Acto quirúrgico & Exodoncia: 35 \\
relacionado & Cirugía de implantes: 7 \\
& Alveoloplastia: 1 \\
\hline Localización de las & Mandibular: 25 \\
lesiones & Maxilar superior: 8 \\
& Maxilo-mandibular: 3 \\
& No evaluable: 7 \\
\hline Estadio de las lesiones & $0: 8$ \\
& I: 8 \\
& II: 9 \\
& III: 2 \\
& No evaluable: 16 \\
\hline
\end{tabular}

\section{DISCUSIÓN}

Aunque la incidencia de la OMB es baja, su importancia está en relación con la morbilidad asociada a este tipo de lesión. Su manejo una vez instaurada es complejo debido a la alta resistencia que presenta al tratamiento. Por ello, el análisis de los factores que de algún modo pueden predisponer o aumentar el riesgo de su aparición es fundamental.
El nivel de evidencia científica derivado de la literatura es bajo, debido en parte al tipo de diseño, ya que se trata de series de casos en su mayor parte. Por otro lado, el registro de datos a menudo es incompleto, en factores considerados de riesgo tales como la dosis o duración del tratamiento ${ }^{5}$

Se han reconocido factores de riesgo derivados tanto de las características basales del paciente, como dependientes del propio tratamiento farmacológico.

En relación a los datos obtenidos sobre los 43 casos estudiados, aunque el sexo, según otros trabajos publicados ${ }^{5}$, no parece determinar un aumento de riesgo para la aparición de $\mathrm{OMB}$, se ha observado que en los casos analizados, existe una clara predilección por sexo femenino. Este hallazgo puede ser debido a que la incidencia de la osteoporosis es mayor en mujeres ${ }^{32,33} \mathrm{y}$, como consecuencia, también es mayor el número de pacientes tratadas con $\mathrm{BP}$ por este motivo.

La edad media de los pacientes registrados en este estudio es elevada (71,94 años).En el contexto de la osteoporosis, la edad juega un papel determinante, ya que se asocia a una pérdida progresiva de la masa ósea ${ }^{32}$ y en mujeres a partir de los 50 años, la prevalencia de esta enfermedad aumenta progresivamente ${ }^{34}$

En cuanto al fármaco empleado, el alendronato ha sido el fármaco implicado en la mayoría de los casos. En nuestro medio parece ser el fármaco prescrito con mayor frecuencia ${ }^{35}$, y existen guías que recomiendan su elección como primera opción de tratamiento ${ }^{36}$ por lo que además de las características del propio fármaco, tales como su potencia, dosificación o vía de administración, podrían influir otros parámetros derivados de su prescripción.

En lo que se refiere a la vía de administración, la $\mathrm{AAOMS}^{5}$ reconoce que el riesgo de OMB asociado al empleo de BP por vía oral, parece incrementarse cuando la duración del tratamiento excede los tres años. El documento publicado por la Sociedad Española de Cirugía Oral y Maxilofacial ${ }^{9}$, expone que el tiempo medio de tratamiento previo a la aparición de esta patología suele ser alrededor de 67,2 meses (5,6 años). 
Otros trabajos informan sobre el hecho de que este período puede ser menor, siendo más frecuente su aparición por encima de los 48 meses de tratamiento ${ }^{4,37}$ En la muestra de casos analizada, la duración media de tratamiento fue inferior (48,25 meses).

Por otro lado, es conocido, que los fármacos administrados por vía intravenosa, tienen un peso mayor en el riesgo de desarrollar OMB con respecto a las formulaciones orales. Esto puede ser debido a que los fármacos de mayor potencia suelen ser administrados por esta vía (ácido zoledrónico) y a que se estima que más del $50 \%$ de dosis intravenosa de BP está biodisponible para su incorporación en la matriz ósea, en comparación de menos del 1\% del BP oral ${ }^{3}$ En este sentido, deben ser considerados otros factores como la frecuencia de dosificación y la potencia del fármaco. Fármacos intravenosos se están empleando a dosis más bajas y con una frecuencia menor a la utilizada habitualmente en el tratamiento de pacientes oncológicos, en el contexto de la osteoporosis, equiparando el riesgo de OMB al de los preparados orales de menor potencia $^{38}$ Sin embargo, por otro lado, hay estudios que apoyan la idea de que, tras cinco años de tratamiento con alendronato, la suspensión del fármaco no aumenta el riesgo de fracturas, y esta medida podría ser considerada para reducir las complicaciones de la OMB en estos pacientes ${ }^{39}$

La localización de las lesiones de forma predominante a nivel mandibular es coincidente con la mayoría de los estudios ${ }^{3}$

En cuanto a las manifestaciones clínicas hay autores que señalan que los casos asociados al tratamiento administrado por vía oral son menos graves y con un curso evolutivo más favorable ${ }^{11,40}$

No todos los casos incluidos en esta revisión permitieron establecer el estadio clínico de las lesiones y en muchos casos, tampoco se informó sobre la persistencia o remisión de las mismas. En los casos que aportaban estos datos, se ha observado que las lesiones más graves (estadio III), se presentan con menor frecuencia.

Se ha reconocido la importancia del componente infeccioso en el desarrollo de la $\mathrm{OMB}^{2}$. Por otro lado, el procedimiento quirúrgico empleado, abre una solución de continuidad que permite la penetración y colonización bacteriana a nivel de oral.En todos los casos que aportaban datos referentes a estudios microbiológicos ${ }^{18,22,24}$ este factor estaba presente. Entre estos agentes patógenos toma especial relevancia el Actinomyces $^{41-44}$ (detectado en todos los casos excepto en uno), que habitualmente forma parte de la flora oral y no produce patología mientras permanece en la superficie de la mucosa. Algunos autores apoyan la teoría de que la presencia de BP a nivel óseo en ambos maxilares, favorece el asentamiento de Actinomyces ${ }^{42,45}$ Una vez instaurada la infección requiere de tratamientos antimicrobianos prolongados para su resolución (entre 3-12 meses).

Marx y $\operatorname{col}^{46}$ propusieron la utilización del CTXs, como predictor de riesgo de la aparición de OMB, aconsejando su utilización como herramienta para la toma de decisiones en procedimientos quirúrgicos orales. De este modo, valores inferiores a $100 \mathrm{pg} / \mathrm{ml}$ se consideran de alto riesgo, entre $100-150 \mathrm{pg} / \mathrm{ml}$ de riesgo moderado y valores superiores a $150 \mathrm{pg} / \mathrm{ml}$ se identificaban como de riesgo mínimo. Otros autores ${ }^{21}$ proponen aumentar el valor propuesto por Marx a $200 \mathrm{pg} / \mathrm{ml}$ para considerar como bajo el riesgo de desarrollo de OMB. Plantean además, la suspensión del BP hasta recuperar valores que superen los 150$200 \mathrm{pg} / \mathrm{ml}$, considerando que se produce un aumento de aproximadamente $2,5 \mathrm{pg} /$ $\mathrm{ml}$ por mes de descanso.

$\mathrm{Al}$ igual que Kunchur y $\mathrm{col}^{21}$, otro estudio ${ }^{47}$ enfatiza en la idea de que esta prueba debe ser empleada para identificar a personas en riesgo, pero no como indicador definitivo en cuanto a la predicción del desarrollo de la OMB. El trabajo de Kwon y col $^{26}$ et al determinó la existencia de una correlación positiva entre la gravedad de las lesiones y la evaluación del riesgo utilizando los valores de CTXs. Su empleo por otro lado, ha sido cuestionado por otros autores y no avalan su utilización con este propósito ${ }^{48,49}$, ya que valores inferiores a $150 \mathrm{pg} / \mathrm{ml}$ son considerados índices de la eficacia terapéutica de los BP. Además es una prueba que aporta valores cuya varia- 
bilidad depende de factores tales como la edad, sexo, consumo de alcohol, tabaco, ejercicio, ritmos circadianos, tipo de test empleado, etc.

Los casos registrados en este documento que aportaban datos a este respecto ${ }^{21,26}$, presentaron en su mayoría valores inferiores a $150 \mathrm{pg} / \mathrm{ml}$ y en sólo tres de ellos los valores fueron superiores a $200 \mathrm{pg} / \mathrm{ml}$. Por tanto, sería preciso realizar investigaciones más amplias, que puedan validar el empleo en la práctica clínica de este marcador como predictor de riesgo de la OMB. Otros autores ${ }^{47}$, abren la puerta hacia la utilización de otro tipo de marcadores como la fosfatasa alcalina sérica.

Aunque las pautas de tratamiento, no fueron objeto de evaluación en esta revisión, llama la atención el interés de tres de las publicaciones incluidas ${ }^{18,27,29}$ sobre el teriparatide como posible agente responsable de la remisión de las lesiones. Este fármaco es otra de las opciones terapéuticas empleadas para el tratamiento de la osteoporosis. Se trata de un importante mediador de la remodelación ósea y se ha reconocido su utilidad en la reducción del riesgo de nuevas fracturas gracias a mecanismos osteoformadores ${ }^{50}$ Serían precisos estudios adicionales para confirmar las hipótesis planteadas por estos autores, evaluando su papel y determinando en que medida contribuyen en la resolución de las lesiones.

Sería de interés estandarizar el registro de datos, incluso desde el inicio de la terapia con BP, con el objetivo de que el análisis de los elementos que puedan ser considerados de relevancia, se realice de forma rigurosa y rutinaria. De este modo, se facilitaría la realización de estudios para conocer la prevalencia real de esta patología y el grado en el que están involucrados los factores de riesgo considerados hasta el momento ${ }^{12,51,52}$.

Dicho registro debería ser llevado a cabo por los distintos facultativos implicados en el tratamiento del paciente osteoporótico, ya que a menudo, los datos aportados por el propio paciente no son un fiel reflejo de la realidad. Desde esta perspectiva,es fundamental la colabora- ción y la comunicación fluida entre todos los profesionales y la educación para la salud, desde el punto de vista de que el paciente debe ser informado sobre el tipo de fármaco que está recibiendo y de la importancia de mantener una adecuada salud bucodental. Este seguimiento, de forma preventiva, minimizaría el riesgo de aparición de OMB.

Son necesarios más estudios prospectivos y retrospectivos, con un diseño adecuado, para evaluar el peso real de cada uno de los factores considerados de riesgo en el desarrollo de la OMB, ya que por cuestiones éticas la realización de ensayos clínicos al respecto sería más que cuestionable. Dada la baja prevalencia de esta complicación, sería preciso realizar estudios multicéntricos para obtener una muestra significativa de casos.

Por otro lado, la revisión realizada, pone de manifiesto que no existe suficiente evidencia científica que avale las actuales recomendaciones clínicas. Estas recomendaciones se basan en opiniones de expertos tanto para el tratamiento de la OMB una vez instaurada, como para la aplicación de estrategias preventivas, en pacientes subsidiarios de cirugía dentoalveolar.

\section{BIBLIOGRAFÍA}

1. NIH Consensus development panel osteoporosis prevention, diagnosis, and therapy. JAMA 2001; 285: 785-795.

2. Muñoz-Torres M, Jódar Gimeno E. Grupo de trabajo de metabolismo mineral óseo de la Sociedad Española de Endocrinología y Nutrición (SEEN). Osteoporosis: informe del grupo de trabajo de metabolismo mineral óseo de la SEEN. Endocrinol Nutr 2007; 54: 53-61.

3. Pazianas M, Miller P, Blumentals Wa, Bernal M, Kothawala P. A review of the literature on osteonecrosis of the jaw in patients with osteoporosis treated with oral bisphosphonates: prevalence, risk factors, and clinical characteristics. Clin Ther 2007; 29:1548-1558.

4. Division of Reproductive and Urologic Products, Office of New Drugs Division of Pharmacovigilance II, Office of Surveillance and Epidemiology Division of Epidemiology, Office of Surveillance and Epidemiology. Center for Drug Evaluation and Research Food and 
Drug Administration. Background Document for Meeting of Advisory Committee for Reproductive Health Drugs and Drug Safety and Risk Management Advisory Committee. Silver Spring, Maryland:FDA, 2011 [acceso 29 de octubre de 2012] Disponible en: http:// www.fda.gov/downloads/AdvisoryCommittees/CommitteesMeetingMaterials/ drugs/DrugSafetyandRiskManagementAdvisoryCommittee/ucm270958.pdf

5. Ruggiero SL, Dodson tB, Assael LA, Landesberg R, Marx RE, Mehrotra B. American Association of Oral and Maxillofacial Surgeons position paper on bisphosphonate-related osteonecrosis of the jaw-2009 update. J Oral Maxillofac Surg 2009; 67 (5 Suppl.):2-12.

6. MARX RE. Pamidronate (Aredia) and zoledronate (Zometa) induced avascular necrosis of the jaws: a growing epidemic. J Oral Maxillofac Surg 2003; 61: 1115-1117.

7. Rugiero SL, Mehrotra B, RosenbergtJ, Engroff SL. Osteonecrosis of the jaws associated with the use of bisphosphonates: a review of 63 cases. J Oral Maxillofac Surg 2004; 62: 527534.

8. Sosa Henríquez M, Gómez de Tejada Romero MJ, Bagán Sebastián JV, Díaz M, Díez Pérez A, Jodar Gimeno $\mathrm{E}$ et al. Osteonecrosis de los maxilares: documento de consenso de la sociedad española de investigación ósea y del metabolismo mineral (SEIOMM). Rev Osteoporos Metab Miner 2009; 1: 41-51.

9. Junquera LM, Martín-Granizo R. Diagnóstico, prevención y tratamiento de la osteonecrosis de los maxilares por bisfosfonatos. Recomendaciones de la sociedad Española de Cirugía Oral y Maxilofacial (SECOM). Rev Esp Cir Oral y Maxilofac 2008; 30: 145-156.

10. Khosla S, Burr D, Cauley J, Dempster DW, EbeLing FR, Felsenberg D, Gagel RS et al. Bisphosphonate-associated osteonecrosis of the jaw: report of a task force of the American Society for Bone and Mineral Research. J Bone Miner Res 2007; 22:1479-1491.

11. Mavrokokki T, Cheng A, Stein B, Goss A. Nature and frecuency of bisphosphonate-associated osteonecrosis of the jaw in Australia. J Oral Maxillofac Surg 2007; 65: 415-423.

12. Bagán J, Blade J, Cozar JM, Constela M, GarcíaSanz R, Gómez-Veiga F et al. Recommendations for the prevention, diagnosis, and treatment of osteonecrosis of the jaw (ONJ) in cancer patients treated with bisphosphonates. Med Oral Patol Oral Cir Bucal 2007; 12: E 336-340.

13. Khan AA, SÁndor GKB, Dore E, Morrison AD, AsahLi M, Amin F et al. Canadian consensus practice guidelines for Biphosphonate asso- ciated osteonecrosis of the jaw. J Rheumatol 2008; 35: 1391-1397.

14. Edwards BJ, Hellstein JW, Jacobsen PL, Kaltman S, Mariotti A, Migliorati CA. Updated recommendations for managing the care of patients receiving oral bisphosphonate therapy: An advisory statement from the American Dental Association Council on Scientific Affairs. J Am Dent Assoc 2008; 139: 1674-1677.

15. Centre for Oral Health Strategy. Prevention of osteonecrosis of the jaw (ONJ) in patients on bisphosphonate therapies. [Internet] Sydney; Centre for Oral HealthStrategy [Elaborado en julio 2010/ consultado enero 2012] Disponible en: http://www.health.nsw. gov.au/policies/gl/2010/pdf/GL2010_010.pdf

16. Cardona F, Bagán JV, SÁinz E, Figuerido J, Giner F, VIDÁN FJ. Osteonecrosis de los maxilares por bisfosfonatos. Actualización y puesta al día. An Sist Sanit Navar 2009; 32: 413-421.

17. Manterola C, Zavando D. Grupo MinCiR. Cómo interpretar los "niveles de evidencia" en los diferentes escenarios clínicos. Rev Chilena de Cirugía 2009; 61:582-595.

18. HARPER RP, Fung E. Resolution of bisphosphonate-associated osteonecrosis of the mandible: possible application for intermittent lowdose parathyroid hormone [rhPTH(1-34)]. J Oral Maxillofac Surg 2007; 65: 573-580.

19. Kumar SKS, Meru MC, Sedghizadeh PP. Osteonecrosis of the jaws secondary to bisphosphonate therapy: a case series. J Contemp Dent Pract 2008; 9: 63-69.

20. FANTASIA JE. Bisphosphonates-what the dentist needs to know: practical considerations. J Oral Maxillofac Surg 2009; 67 (5 Suppl): 5360.

21. Kunchur R, Need A, Hughes T, Goss A. Clinical investigation of $\mathrm{C}$-terminal cross-linking telopeptide test in prevention and management of bisphosphonate-associated osteonecrosis of the jaws. J Oral Maxillofac Surg 2009; 67: 1167-1173.

22. Alons K, KujJPers SC, de Jong E, van MerKesTEYN JP. Treating low- and medium-potency bisphosphonate-related osteonecrosis of the jaws with a protocol for the treatment of chronic suppurative osteomyelitis: report of 7 cases. Oral Surg Oral Med Oral Pathol Oral Radiol Endod 2009; 107:e1-7.

23. Yamaguchi K, Ozzumi T, Funayama H, Kawamura $\mathrm{H}$, Sugawara S. Osteonecrosis of the jawbones in 2 osteoporosis patients treated with nitrogen-containing bisphosphonates: osteonecrosis reduction replacing NBP with non-NBP (etidronate) and rationale. J Oral Maxillofac Surg 2010; 68: 889-897. 
24. Dannemann C, Gratz KW, Riener MO, Zwahlen RA. Jaw osteonecrosis related to bisphosphonate therapy: A severe secondary disorder. Bone 2007; 40: 828-834.

25. Hutchinson M, O'Ryan F, Chavez V, Lathon PV, SAnchez G, Hatcher DC et al. Radiographic findings in bisphosphonate-treated patients with stage 0 disease in the absence of bone exposure. J Oral Maxillofac Surg 2010; 68: 2232-2240.

26. Kwon YD, Kim DY, Ohe JY, Yoo JY, Walter C. Correlation between serum C-terminal crosslinking telopeptide of type I collagen and staging of oral bisphosphonate-related osteonecrosis of the jaws. J Oral Maxillofac Surg 2009; 67: 2644-2648.

27. Subramanian G, Cohen HV, Quek SYP. A model for the pathogenesis of bisphosphonateassociated osteonecrosis of the jaw and teriparatide's potential role in its resolution. Oral Surg Oral Med Oral Pathol Oral Radiol Endod 2011; 112: 744-753.

28. Treister NS, Friedland B, Woo SB. Use of conebeam computerized tomography for evaluation of bisphosphonate-associated osteonecrosis of the jaws. Oral Surg Oral Med Oral Pathol Oral Radiol Endod 2010; 109: 753-764.

29. Narongroeknawin P, Danila MI, Humphreys LG, Barasch A, Curtis JR. Bisphosphonate-associated osteonecrosis of the jaw, with healing after teriparatide: a review of the literature and a case report. Spec Care Dentist 2010; 30: 77-82.

30. Bedogni A, Bettini G, Totola A, Saia G, Nocini PF. Oral bisphosphonate-associated osteonecrosis of the jaw after implant surgery: a case report and literature review. J Oral Maxillofac Surg 2010; 68: 1662-1666.

31. Goss A, Bartold M, Sambrook P, Hawker P. The nature and frequency of bisphosphonate-associated osteonecrosis of the jaws in dental implant patients: a south australian case series. J Oral Maxillofac Surg 2010; 68: 337-343.

32. Montero M, Calabuig E, Muñoz ML, Valero JL, Todolí J, CAlabuig JR et al. Densitometría axial y periférica en el diagnóstico de la osteoporosis. Reemo 2002; 11: 140-143.

33. González Macías J, Riancho Moral J. Osteoporosis. Concepto. Epidemiología. Etiología. Manifestaciones clínicas y complicaciones. Medicine 2006; 9: 3873-3879.

34. Grova Marco MV, García Santana S, Miravalle Pescador A, González Rodríguez E, González PAdilla E, Sosa Henríquez M. Enfermedades metabólicas óseas. Introducción. Remodelado óseo y pérdida ósea. Osteoporosis: concepto, epidemiología, morbilidad y mortalidad.
Repercusiones económicas de la osteoporosis. Enfermedad infradiagnosticada. Medicine 2010; 10: 4109-4116.

35. Carbonell C, Díez A, Calaf J, Caloto MT, Nocea G, LARA N. Pauta de tratamiento inicial en pacientes con osteoporosis: uso de antirresortivos y suplementos farmacológicos (calcio y vitamina D) en la práctica clínica. Reumatol Clin 2012; 8: 3-9.

36. Alendronate, etidronato, risedronato, raloxifene, strontium ranelate and teriparatide for the secondary prevention of osteoporotic fragility fractures in postmenopausal women.[Internet]. London. NICE. 2005 [Elaborado en 2008/ consultado en mayo de 2012]. Technology Appraisal Guidance 161. Disponible en: http://www.nice.org.uk/TA161

37. Palaska PK, Cartsos V, Zavras AI. Bisphosphonates and time to osteonecrosis developement. Oncologist 2009; 14: 1154-1166.

38. Black DM, Delmas PD, Eastell R, Reid IR, Boonen S, CAuley JA et al. Once-yearly zoledronic acid for treatment of postmenopausal osteoporosis. N Engl J Med 2007; 356: 1809-1822.

39. Black DM, Schwartz AV, Ensrud KE, Cauley JA, Levis S, QuANDT SA et al. Effects of continuing or stopping alendronate after 5 years of treatment. The fracture intervention trial long-term extension (FLEX): a randomized trial. JAMA 2006; 296: 2927-2938.

40. Cardona Tortajada F, Sainz Gómez E, Giner MuÑOZ F, Figuerido Garmendia J. Osteonecrosis de los maxilares por bisfosfonatos orales. Presentación de 3 casos. Aten Primaria 2012; 44 : 116-117.

41. Kaplan I, Anavi K, Anavi Y, Calderon S, SchwartzArad D, Teicher $\mathrm{S}$ et al. The clinical spectrum of Actinomyces-associated lesions of the oral mucosa and jawbones: correlations with histomorphometric analysis. Oral Surg Oral Med Oral Pathol Oral Radiol Endod 2009; 108: 738-746.

42. NAIK NH, Russo TA. Bisphosphonate-related osteonecrosis of the jaw: the role of Actinomyces. Clin Infect Dis 2009; 49: 1729-1732.

43. LEE CYS, PIEn FD, SuZuKI JB. Identification and treatment of bisphosphonate-associated actinomycotic osteonecrosis of the jaws. Implant Dentistry 2011; 20: 331-336.

44. Hansen T, Kunkel M, Springer E, Walter C, Weber A, Siegel E et al. Actinomycosis of the jawshistopathological study of 45 patients shows significant involvement in bisphosphonateassociated osteonecrosis and infected osteoradionecrosis. Virchows Arch 2007; 451: 1009-1017. 
45. Kos M, Brusco D, Kuebler J, EngelKe W. Clinical comparison of patients with osteonecrosis of the jaws, with and without a history of bisphosphonates administration. Int J Oral Maxillofac Surg 2010; 39: 1097-1102.

46. Marx RE, Cillo JE JR, UlloA JJ. Oral bisphosphonate-induced osteonecrosis: risk factors, prediction of risk using serum CTX testing, prevention, and treatment. J Oral Maxillofac Surg 2007; 65: 2397-2410.

47. Lazarovici TS, Mesilaty-Gross S, Vered I, Pariente C, Kanety H, Givol N et al. Serologic bone markers for predicting development of osteonecrosis of the jaw in patients receiving bisphosphonates. J Oral Maxillofac Surg 2010; 68: 2241-2247.

48. American Society for Bone and Mineral Research Task Force on Osteonecrosis of the Jaw. Khosla S, Burr D, Cauley J, Dempster DW, Ebeling PR, Felsenberg D et al. Oral bisphos- phonate-induced osteonecrosis: risk factors, prediction of risk using serum CTX testing, prevention, and treatment. J Oral Maxillofac Surg 2008; 66: 1320-1321.

49. LEE CYS, SuZUKI JB. CTX biochemical marker of bone metabolism. Is it a reliable predictor of bisphosphonate-associated osteonecrosis of the jaws after surgery? Part II: A prospective clinical study. Implant Dentistry 2010; 19:29-38.

50. Sosa Henríquez M, Díez Pérez A. La hormona paratiroidea en el tratamiento de la osteoporosis. An Med Interna 2007; 24: 87-97.

51. GlikLich R, Wilson J. Epidemiology of bisphosphonate-related osteonecrosis of the jaws: The utility of a national registry. J Oral Maxillofac Surg 2009; 67: 71-74.

52. Ribas-Deix O, SAnz-Gallén P, Nogué S. Osteonecrosis de la mandíbula asociada al tratamiento oral con ibandronato. An Sist Sanit Navar 2011; 34: 489-491. 
